\title{
Systematic Evaluation of Phantom Fluids for Simultaneous PET/MR Hybrid Imaging
}

\author{
Susanne Ziegler ${ }^{1}$, Harald Braun ${ }^{1}$, Philipp Ritt ${ }^{2,3}$, Carsten Hocke$^{2}$, Torsten Kuwert ${ }^{2}$, and Harald H. Quick ${ }^{1}$ \\ ${ }^{1}$ Institute of Medical Physics, University of Erlangen-Nuremberg, Erlangen, Germany; ${ }^{2}$ Clinic for Nuclear Medicine, University \\ Hospital Erlangen, Erlangen, Germany; and ${ }^{3}$ Pattern Recognition Lab, University of Erlangen-Nuremberg, Erlangen, Germany
}

\begin{abstract}
With the recent advent of integrated PET/MR hybrid systems, the need for simultaneous PET and MR phantom measurements arises. Phantom fluids that are used in stand-alone MR systems, especially in larger phantoms and at a high magnetic field strength, are not necessarily applicable in PET imaging and vice versa. In this study, different approaches to fluid selection were considered and systematically evaluated with respect to their usability for simultaneous PET/MR phantom imaging. Methods: Demineralized water, water with increased electrical conductivity, a water-oil emulsion, and monoethylene and triethylene glycol were investigated in MR and PET measurements using the most common PET tracer ${ }^{18} \mathrm{~F}$ FDG. As an alternative to ${ }^{18} \mathrm{~F}-\mathrm{FDG}$, a modified PET tracer $\left({ }^{18} \mathrm{~F}\right.$-fluoride Kryptofix 222 complex) was investigated toward its ability to dissolve in pure oil, which provides good signal homogeneity in MR imaging. Measurements were performed on a 3.0 T integrated PET/ MR whole-body system using a National Electrical Manufacturers Association quality-standard phantom. Results: All tested fluids dissolved the radiotracer ${ }^{18} \mathrm{~F}-\mathrm{FDG}$ homogeneously. Regarding their suitability for MR at 3.0 T, all fluids significantly improved the homogeneity compared to pure water (increase of excitation flip angle within the tested phantom by a factor of 2.0). When the use of ${ }^{18} \mathrm{~F}-$ FDG was preferred, triethylene glycol provided the best compromise (flip angle increase by a factor of 1.13). The potential alternative tracer, ${ }^{18} \mathrm{~F}$-fluoride Kryptofix 222 complex, dissolved in pure oil; however, it is not optimal in its tested composition because it accumulates at the bottom of the phantom during the time of measurement. Conclusion: This study provides a systematic approach toward phantom fluid selection for imaging a given quality-standard body phantom - and phantoms of comparable size-at 3.0 T. For simultaneous PET/MR scans using the standard tracer ${ }^{18} \mathrm{~F}-\mathrm{FDG}$, an alternative fluid to water and oil is proposed that serves as a viable option for both imaging modalities. Nevertheless, when water is preferred, ways to improve MR image homogeneity are presented. The tested alternative PET tracer enables the use of pure oil in combined scans, but the tracer composition needs to be optimized for phantom measurement applications.
\end{abstract}

Key Words: integrated PET/MR hybrid imaging; PET/MR phantom measurements; NEMA standard phantom; radiofrequency artifact; inhomogeneous radiofrequency excitation

J Nucl Med 2013; 54:1464-1471

DOI: 10.2967/jnumed.112.116376

Received Oct. 26, 2012; revision accepted Feb. 7, 2013.

For correspondence or reprints contact: Susanne Ziegler, Institute of Medical Physics (IMP), University of Erlangen-Nuremberg, Henkestrasse 91, 91052 Erlangen, Germany.

E-mail: susanne.ziegler@imp.uni-erlangen.de

Published online Jun. 21, 2013.

COPYRIGHT (C) 2013 by the Society of Nuclear Medicine and Molecular Imaging, Inc.
I n medical imaging, phantom measurements are a necessity for quality-control purposes of the scanner hardware and software and for evaluating newly developed algorithms and methods. With the recent introduction of sequential and integrated PET/MR hybrid scanners, the need for combined and simultaneous MR and PET phantom measurements arises $(1,2)$. However, phantom fluids that are used in separate PET or MR imaging may not be interchangeably applicable.

In both imaging modalities, phantoms usually consist of plastic containers filled with signal-providing liquid. In PET imaging, pure water is used in phantom inserts and in the surrounding background fluid because it can dissolve the most commonly used radioactive tracer, ${ }^{18}$ F-FDG, well. However, water may induce strong artifacts in MR imaging when scanning large phantoms (Fig. 1A) at a high magnetic field strength (above $1.5 \mathrm{~T}$ ). These artifacts are caused by inhomogeneous radiofrequency $(\mathrm{RF})$ excitation when fluids with high relative permittivity $\varepsilon_{\mathrm{r}}$, such as water $\left(\varepsilon_{\mathrm{r}}=76\right.$, Table 1$)$, are imaged (Fig. 1B) (3-12). Thus, in those cases oil-based substances are preferred as MR phantom fillers because of the low $\varepsilon_{\mathrm{r}}$ of oil $\left(\varepsilon_{\mathrm{r}}\right.$ $=2.7$, Table 1). However, oil-based substances are in turn not applicable for PET imaging because they do not dissolve the tracer ${ }^{18}$ F-FDG (Fig. 1C).

In addition to the $\varepsilon_{\mathrm{r}}$ of a substance, the electrical conductivity $\sigma$ of the medium also contributes to the distribution of the RF field. An increase in the $\sigma$ causes the RF field to be attenuated toward the center of the sample, and the aforementioned RF artifacts are significantly reduced (7-9). Consequently, 2 approaches for avoiding these artifacts can be pursued: either using alternative substances with decreased $\varepsilon_{\mathrm{r}}$, compared to water, or increasing the conductivity of water. However, for the 2nd approach the extent to which the inhomogeneous RF excitation (in the present coil-phantom setup resulting in an RF increase toward the phantom center) is compensated by additional higher conductivity needs to be evaluated.

As a 3rd approach, instead of searching for an appropriate solvent for ${ }^{18} \mathrm{~F}$-FDG, one can consider using a different tracer that, as opposed to ${ }^{18} \mathrm{~F}-\mathrm{FDG}$, dissolves in pure oil.

The goal of this study was therefore to evaluate phantom fluids and alternative tracers with regard to their usability in both MR and PET imaging in larger phantoms. Aiming toward homogeneous MR and PET signal across large phantoms will potentially allow for simultaneous and time-efficient measurements of PET and MR image quality parameters or will improve MR-based attenuation correction (AC) of PET phantom measurements. 


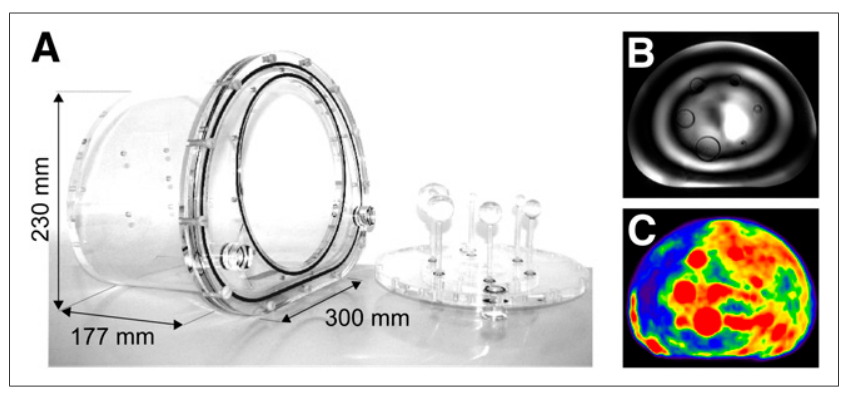

FIGURE 1. International Electrotechnical Commission standard 61675-1 body phantom investigated in this study (A) (14). Images of inhomogeneous MR excitation in water (B) and inhomogeneous PET tracer distribution in oil-based substances (C).

\section{MATERIALS AND METHODS}

\section{PET/MR Hybrid Scanner}

Measurements were performed on an integrated PET/MR wholebody hybrid system (Biograph mMR; Siemens AG Healthcare Sector), which allows for simultaneous PET and MR imaging.

The hybrid system consists of an actively shielded 3.0 T magnet and a gradient coil unit characterized by a maximum amplitude of 45 $\mathrm{mT} / \mathrm{m}$ and a maximum slew rate of $200 \mathrm{~T} / \mathrm{m} / \mathrm{s}$. RF excitation is performed using a single-channel quadrature RF body coil.

The PET detector unit is completely integrated into the hybrid system and is located between the MR gradient coil unit and the RF body coil inside the scanner. One PET detector block contains $8 \times 8$ lutetium oxyorthosilicate scintillator crystal elements and uses an array of $3 \times 3$ avalanche photodiodes (13). Eight detector rings form the PET detector unit, each consisting of 56 detector blocks.

\section{Phantom}

Phantom fluids are evaluated in a body-mimicking phantom (PTW) conformable to standard 61675-1 of the International Electrotechnical Commission (Fig. 1A). This phantom is commonly used in PET imaging and is part of image quality tests according to the standard of the National Electrical Manufacturers Association $(14,15)$.

The dimensions of the phantom housing are given in Figure 1. According to the manufacturer's specification, the phantom body is built of acrylic glass material and has a volume of $9.6 \mathrm{~L}$ when no inserts are used. The insert features 6 hollow glass spheres with different inner diameters $(10,13,17,22,28$, and $37 \mathrm{~mm})$. These can be filled with fluid and PET tracer separately from the large surrounding

TABLE 1

Parameters of Chosen Fluids

\begin{tabular}{lcc}
\hline \multicolumn{1}{c}{ Medium } & $\varepsilon_{\mathrm{r}}$ & $\sigma$ in $(\mathrm{S} / \mathrm{m})$ \\
\hline Water (demineralized) & 75.68 & 0.003 \\
Water $(1 \mathrm{~L})+3.75 \mathrm{~g}$ of $\mathrm{NiSO}_{4}$ & 77.57 & 0.192 \\
Water $(1 \mathrm{~L})+3.75 \mathrm{~g}$ of & 72.84 & 1.109 \\
NiSO $4+5 \mathrm{~g}$ of $\mathrm{NaCl}$ & & \\
Monoethylene glycol $_{\text {Triethylene glycol }}$ & 39.41 & 0.022 \\
Emulsion $(75 \%$ oil, 25\% water) & 21.66 & 0.020 \\
Oil & 12.38 & 0.007 \\
& 2.68 & 0.001 \\
\hline
\end{tabular}

Measurements were performed at resonance frequency of $125.5 \mathrm{MHz}(\sim$ Larmor frequency at $3 \mathrm{~T})$ at $23^{\circ} \mathrm{C}\left(73.4^{\circ} \mathrm{F}\right)$. Substances are listed according to decreasing $\varepsilon_{\mathrm{r}}$ from water to oil. compartment and thus simulate different lesion sizes and lesion-tobackground activity ratios.

\section{Phantom Fluid Selection: Overview}

Figure 2 describes the approaches of phantom fluid selection that were investigated in this study. If the standard PET tracer ${ }^{18} \mathrm{~F}-\mathrm{FDG}$ or other polar tracers are preferred, polar phantom fluids are necessary. Similarly, for nonpolar oil as a phantom fluid, nonpolar PET tracers are required. Because these nonpolar tracers are in general not commercially available, the use of crown ethers as phase-transfer catalysts was investigated.

Concerning phantom fluid suitability for MR measurements, the goal of this study was to obtain artifact-free images throughout a wide range of MR imaging sequences. Therefore, artifacts originating from inhomogeneous RF excitation or T1 relaxation artifacts are to be minimized.

In addition to the aforementioned specific requirements for PET and MR imaging, the compatibility with acrylic glass material is of general importance. Information about acrylic glass compatibility can be obtained from manufacturers.

\section{Fluid Selection for ${ }^{18}$ F-FDG PET}

Pure Fluids as Alternative to Water. ${ }^{18} \mathrm{~F}-\mathrm{FDG}$ is the most commonly used tracer for PET imaging in patients and is therefore frequently used in phantom experiments in clinical routine. Because liquids with reduced $\varepsilon_{\mathrm{r}}$ are less polar and therefore in general less miscible with other polar substances such as ${ }^{18} \mathrm{~F}-\mathrm{FDG}$, the goal of this study was to find a practical compromise between low $\varepsilon_{\mathrm{r}}$ and sufficient ${ }^{18} \mathrm{~F}-\mathrm{FDG}$ miscibility.

Among general solvents, one group of fluids that meets all of the specific requirements mentioned is ethylene glycols. Monoethylene, diethylene, and triethylene glycol are characterized by a significantly lower $\varepsilon_{\mathrm{r}}$ than water (Table 1), and a sufficient resistance of acrylic glass to glycols is reported (16). For investigation in MR and PET measurements, monoethylene and triethylene glycol were chosen.

Emulsions as Alternative to Water. Because of the described characteristics of its individual components, it is evident that a mixture of oil and water would be preferable for PET/MR phantom measurements. Oil is used to reduce the $\varepsilon_{\mathrm{r}}$ and water to dissolve ${ }^{18} \mathrm{~F}-\mathrm{FDG}$. However, oil and water do not mix because of their different polarities (17). To address this problem, an emulsifier was used.

In a preliminary study evaluating various emulsifier and oil-water compositions (data not shown), it was empirically determined that a mixture of $7 \mathrm{~mL}$ of emulsifier-consisting of $62 \%$ polysorbate 80 (CAS registry no. 9005-65-6; Caesar \& Loretz GmbH) and 38\% sorbitan trioleate (CAS registry no. 26266-58-0; Caesar \& Loretz $\mathrm{GmbH}$ ) - per $100 \mathrm{~mL}$ of oil-water emulsion (75\% oil, $25 \%$ water) resulted in the best emulsion composition. In the small samples, the tested emulsion was stable for at least 1 day. In the large volume of the body phantom $(\sim 10 \mathrm{~L})$, the stability decreased significantly to $1-2 \mathrm{~h}$ because of the increased difficulty in emulsion preparation.

Supplementing Water for Artifact Reduction. In addition to water's high $\varepsilon_{\mathrm{r}}$, a further potential source for image artifacts is the long T1 relaxation time of water. In combination with specific imaging parameters (e.g., short repetition time), artifacts may be caused that show patterns different from those that derive solely from high permittivity. Therefore, a shortened $\mathrm{T} 1$ relaxation time, which can be accomplished by adding nickel sulfate $\left(\mathrm{NiSO}_{4}\right)$, is preferable. A T1 time reduction from the initial value of approximately $2600 \mathrm{~ms}$ to $100 \mathrm{~ms}$ was achieved by the addition of $3.75 \mathrm{~g}$ of $\mathrm{NiSO}_{4}$ per liter of demineralized water.

Adding $\mathrm{NiSO}_{4}$ and $\mathrm{NaCl}$ to water increases the electrical conductivity $\sigma$ (Table 1). Empiric investigation with various amounts of $\mathrm{NaCl}$ (data not shown) showed that $5 \mathrm{~g}$ of $\mathrm{NaCl}$ per $1 \mathrm{~L}$ of water- $\mathrm{NiSO}_{4}$ mixture gave the best results, and hence this amount was used. This fluid composition is identical to common MR phantom fluids used by manufacturers in phantom liquid bottles. 


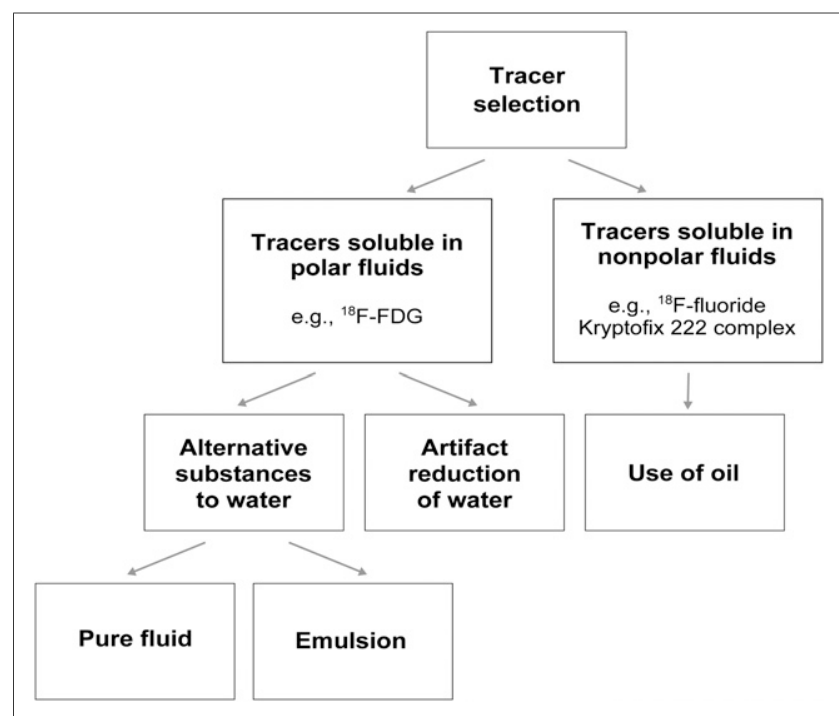

FIGURE 2. Overview over investigated approaches of fluid selection in this study.

\section{Tracer Selection for Use of Oil as Phantom Fluid}

Replacing ${ }^{18} \mathrm{~F}-\mathrm{FDG}$ with a tracer that dissolves in nonpolar oil would provide the best PET/MR imaging conditions. For this purpose, crown ethers could be used. These cyclic polyethers contain a polar region in their cavity and a nonpolar exterior and thus have the ability to cause polar compounds to dissolve in nonpolar, organic solvents. The specific crown ether 18 -crown-6, for example, will bind positively charged potassium ions $\left(\mathrm{K}^{+}\right)$in its cavity and can be used to transfer negatively charged ions (e.g., tracer ${ }^{18} \mathrm{~F}$-fluoride ions) into the organic environment (18). These crown ethers (trade name Kryptofix 222, CAS registry no. 23978-09-8; Merck KGaA) are generally used in the synthesis of ${ }^{18} \mathrm{~F}$-FDG where fluoride anions $\left({ }^{18} \mathrm{~F}^{-}\right)$are eluted with an aqueous acetonitrile solution of Kryptofix 222 and potassium carbonate from an ion exchange column (19-23). Because the linkage with glucose is negligible for phantom measurements, the ${ }^{18} \mathrm{~F}$ decay in this stage provides the same imaging conditions as with ${ }^{18} \mathrm{~F}$-FDG. In this study, this intermediate stage without further processing is eval- uated as a potential tracer in PET/MR phantom measurements. This tracer is termed ${ }^{18} \mathrm{~F}$-fluoride Kryptofix 222 complex.

\section{Parameter Overview of Selected Fluids}

Table 1 gives an overview of the fluids under investigation and their respective $\varepsilon_{\mathrm{r}}$ and $\sigma$.

These values were measured using a calibrated setup consisting of a probe head immersed in a measurement chamber filled with the different fluids. The probe head was connected to a network analyzer (ZVL3; Rohde \& Schwarz GmbH).

\section{PET Data Acquisition}

To analyze whether the tracer used (either ${ }^{18} \mathrm{~F}-\mathrm{FDG}$ or ${ }^{18} \mathrm{~F}$-fluoride Kryptofix 222 complex) dissolved in the selected fluids, a first test with small samples of liquids $(100 \mathrm{~mL})$ was performed on a PET/CT system featuring a 40-slice CT scanner (Biograph mCT; Siemens AG Healthcare Sector). Subsequently, scans were obtained on the Biograph mMR PET/MR system (Siemens AG Healthcare Sector) using the described body phantom (Fig. 1A). Triethylene glycol and the emulsion were selected as phantom background fluid. A scan with water mixed with $\mathrm{NiSO}_{4}$ served as reference. The spheres were filled with water and gadolinium contrast agent (Gadovist; Bayer) in the ratio of 80:1. The tracer was injected into both the spheres and the phantom background fluid in an approximate activity concentration ratio of 8:1. Detailed imaging and reconstruction parameters are specified in Table 2.

The solubility of the ${ }^{18} \mathrm{~F}$-fluoride Kryptofix 222 complex was investigated in samples of oil, water, monoethylene glycol, and triethylene glycol. In the large body phantom, the tracer was injected into pure oil as background fluid into the water-filled inserts. The tracer solution consisted of $15 \mathrm{mg}$ of Kryptofix 222 and $15 \mu \mathrm{L}$ of potassium carbonate, dissolved in $200 \mu \mathrm{L}$ of water and $800 \mu \mathrm{L}$ of acetonitrile.

\section{MR Data Acquisition}

For MR data acquisition, the body phantom was positioned on the spine array RF coil built into the PET/MR hybrid system's patient table. For all tested protocols, 6 of 24 coil elements of the spine array coil were activated for RF signal reception. Additionally, the phantom was covered by a 6-channel RF body matrix coil.

For MR image quality comparison, different standard MR sequences, as specified in Table 3, were scanned for each fluid in the body phantom.

TABLE 2

PET Imaging and Reconstruction Parameters

\begin{tabular}{|c|c|c|c|c|}
\hline \multirow[b]{2}{*}{ Scan parameters } & \multicolumn{2}{|c|}{${ }^{18} \mathrm{~F}-\mathrm{FDG}$} & \multicolumn{2}{|c|}{${ }^{18}$ F-fluoride Kryptofix 222 complex } \\
\hline & 100-mL samples & Body phantom & 100-mL samples & Body phantom \\
\hline $\begin{array}{l}\text { Activity concentration at } \\
\text { scan time (range) }\end{array}$ & $2 \mathrm{MBq}$ & $\begin{array}{c}\text { Bg: 25-38 MBq, } \\
\text { Sph: 1.28-1.32 MBq }\end{array}$ & $4 \mathrm{MBq}$ & $\begin{array}{l}\text { Bg: } 58 \mathrm{MBq} \\
\text { Sph: } 2.3 \mathrm{MBq}\end{array}$ \\
\hline Scan duration (min) & 2 & 60 & 3 & 60 \\
\hline Reconstruction algorithm & $\begin{array}{l}\text { OSEM incorporating point } \\
\text { spread function } \\
\text { (TrueX; Siemens ) }\end{array}$ & OSEM & $\begin{array}{l}\text { OSEM incorporating point } \\
\text { spread function } \\
\text { (TrueX; Siemens) }\end{array}$ & OSEM \\
\hline Iterations & 2 & 3 & 2 & 3 \\
\hline Subsets & 12 & 21 & 12 & 21 \\
\hline Pixel matrix & $200 \times 200$ & $172 \times 172$ & $200 \times 200$ & $172 \times 172$ \\
\hline Pixel size $\left(\mathrm{mm}^{2}\right)$ & $4.07 \times 4.07$ & $4.17 \times 4.17$ & $4.07 \times 4.07$ & $4.17 \times 4.17$ \\
\hline Slice thickness (mm) & 3 & 2.03 & 3 & 2.03 \\
\hline
\end{tabular}

Scans were performed for both $100-\mathrm{mL}$ samples and large body phantom using ${ }^{18} \mathrm{~F}-\mathrm{FDG}$ and ${ }^{18} \mathrm{~F}$-fluoride Kryptofix 222 complex. Ranges of activity concentration at scan time result from measurements of different fluids.

$\mathrm{Bg}=$ background; Sph = spheres; OSEM = ordered subset expectation maximization. 
TABLE 3

MR Imaging Parameters of Measured Sequences

\begin{tabular}{|c|c|c|c|c|c|}
\hline \multirow[b]{2}{*}{ Parameter } & \multicolumn{2}{|c|}{$2 \mathrm{D}$} & \multicolumn{2}{|r|}{$3 \mathrm{D}$} & \multirow[b]{2}{*}{$\mathrm{B}_{1}$ map } \\
\hline & T2 HASTE & T1 FLASH & VIBE & AC Dixon VIBE in-phase & \\
\hline Repetition time/echo time (ms) & $670 / 48$ & $540 / 2.5$ & $3.25 / 1.23$ & $3.6 / 2.46$ & $300 / 14$ \\
\hline Flip angle $\left(^{\circ}\right)$ & 150 & 75 & 8 & 10 & 90 \\
\hline Field of view $\left(\mathrm{mm}^{2}\right)$ & $248 \times 320$ & $261 \times 310$ & $261 \times 310$ & $328 \times 500$ & $310 \times 310$ \\
\hline Matrix size & $320 \times 248$ & $320 \times 270$ & $512 \times 432$ & $192 \times 126$ & $128 \times 128$ \\
\hline Slice thickness (mm) & 4 & 3 & 2 & 3.12 & 10 \\
\hline Echo train length & 256 & 1 & 1 & 1 & 1 \\
\hline
\end{tabular}

HASTE $=$ half Fourier-acquired single-shot turbo spin echo; FLASH = fast low angle shot; VIBE = volume interpolated breathhold examination.

A 3-dimensional Dixon volume interpolated breathhold examination sequence (VIBE) was used for AC of the PET data in PET/MR hybrid imaging (24). Additionally, the RF homogeneity was measured and quantified directly, using a 2-dimensional spin-echo sequence, which generates maps of the spatial distribution of the RF field in the object $\left(B_{1}\right.$ maps). These maps are calculated from the amplitude ratio of stimulated echo and spin echo arising from 3 applied RF pulses with flip angles $\alpha, 2 \alpha, \alpha$.

Demineralized water with $\mathrm{NiSO}_{4}$, water with $\mathrm{NiSO}_{4}$ and $\mathrm{NaCl}$, monoethylene glycol, triethylene glycol, and the emulsion were used as background fluid. The spheres were filled with water and gadolinium contrast agent in the ratio of 80:1.

\section{RESULTS}

\section{${ }^{18}$ F-FDG PET Imaging}

Water and oil served as references for the best and worst ${ }^{18}$ F-FDG miscibility, when ${ }^{18} \mathrm{~F}$-FDG dissolvability was tested in the liquid samples (Fig. 3A). Monoethylene and triethylene glycol and the emulsion dissolved ${ }^{18}$ F-FDG homogeneously.
The tracer distribution in the different fluids for the large body phantom is shown in Figure 3B and was additionally evaluated in Figure 4. Two circular regions of interest (ROIs) were placed in the center of the phantom in transversal and coronal orientation. The ratio of mean activity concentration within each ROI and mean value over all slices within the phantom was calculated and plotted in the corresponding graphs. In the large phantom, the emulsion and triethylene glycol dissolved ${ }^{18} \mathrm{~F}-\mathrm{FDG}$ comparably to water with $\mathrm{NiSO}_{4}$ (Figs. 3B and 4). However, because of the reduced permittivity and polarity, respectively, it is inevitable that the ${ }^{18}$ F-FDG dissolvability is reduced, compared to water. Therefore, more time and mixing effort are required. Concerning the emulsion, air bubbles appeared and accumulated at the top part of the phantom where, consequently, no tracer was present (Fig. $3 \mathrm{~B}$, indicated by the arrow). Furthermore, reduced stability can cause the separation of the emulsion into its water and oil components over time and hence impair longer phantom measurements, as can also be detected in the performed scan (Fig. 4B).
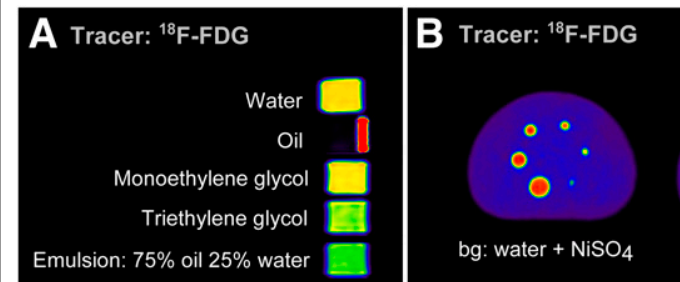

bg: water $+\mathrm{NiSO}_{4}$

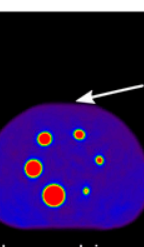

bg: emulsion

bg: triethylene glycol

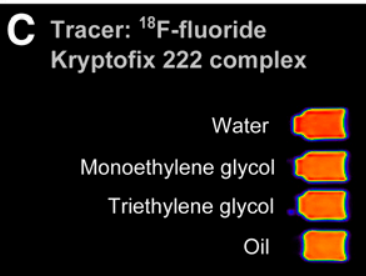

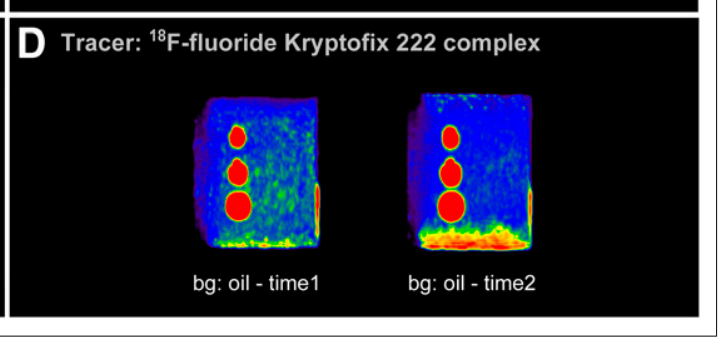

FIGURE 3. PET measurements demonstrating ${ }^{18} \mathrm{~F}-\mathrm{FDG}$ distribution in liquid samples (A); in body phantom with water plus $\mathrm{NiSO}_{4}$, the emulsion, and triethylene glycol as background fluid (B); and the tracer distribution using ${ }^{18} \mathrm{~F}$-fluoride Kryptofix 222 complex as tracer (C and D). ${ }^{18} \mathrm{~F}$-fluoride Kryptofix 222 complex distributed well in all liquid samples (C). Additional tests in body phantom are shown in sagittal view of maximum-intensity projection in images in $\mathrm{D}$. These images show increasing inhomogeneous tracer distribution due to tracer accumulation on bottom of phantom already 20 min after injection (time1). This phenomenon increased over time (3 $\mathrm{h}$ after injection) (time2). bg = background.

\section{${ }^{18}$ F-Fluoride Kryptofix 222 Complex PET Imaging}

${ }^{18}$ F-fluoride Kryptofix 222 complex as a tracer dissolved well in all tested liquid samples (Fig. 3C). In the context of this study, the solubility in pure oil is of significance. At the same time, the additional solubility in water is valuable for PET/ MR phantom measurements because the same tracer could thus be used in inserts filled with water plus in oil as background fluid.

However, when testing this setup in the large body phantom, the tracer composition turned out to be not optimal for phantom measurements (Fig. 3D). The tracer accumulated at the bottom of the phantom over time, already early after tracer injection (Fig. 3D; time1= $20 \mathrm{~min}$ after injection) and increasingly during longer measurements (Fig. 3D; time $2=3 \mathrm{~h}$ after injection). This accumulation can also be observed in Figure 4. Additional mixing efforts between scans did not improve the tracer homogeneity. After the ${ }^{18} \mathrm{~F}$ decay, a small amount of 


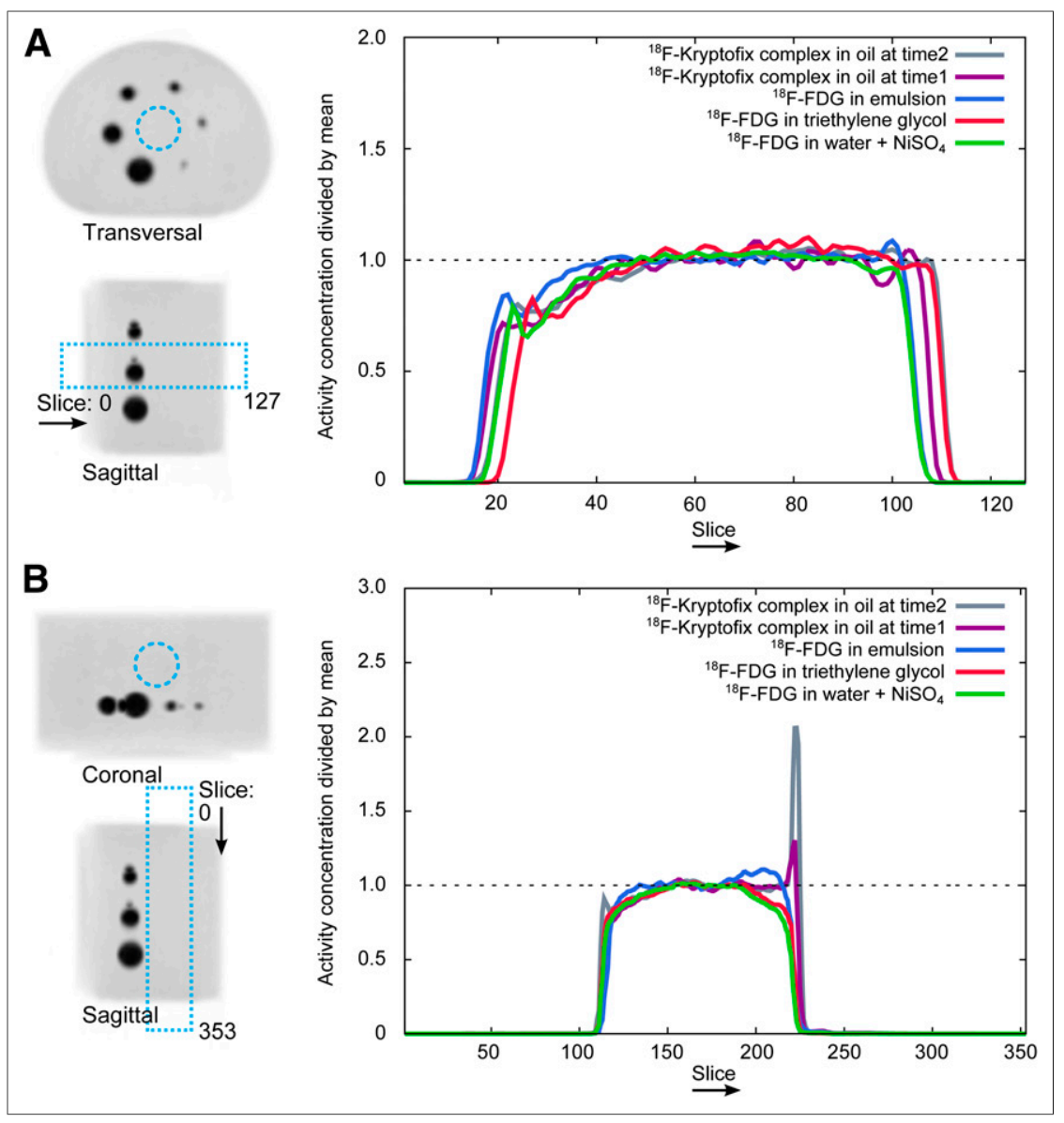

FIGURE 4. Normalized tracer distribution in PET measurements of body phantom in mean of circular ROI (indicated in maximum-intensity-projection images on the left) for each slice in transversal $(\mathrm{A})$ and coronal orientation (B). Both emulsion and triethylene glycol dissolved ${ }^{18} \mathrm{~F}$-FDG comparably to water in phantom. Increase of activity concentration at bottom of phantom in emulsion suggests that emulsion had started to separate into its components during PET scan. Accumulation of tracer at bottom of phantom when ${ }^{18} \mathrm{~F}$ fluoride Kryptofix 222 complex in oil is used (activity peaks of gray and purple line plots) can be observed.

Kryptofix complex remains in the oil, but will not negatively affect subsequent measurements.

\section{MR Imaging}

Figure 5 provides a comprehensive matrix of MR images of the selected fluids measured with the described standard MR protocols. When pure water is scanned, the automatic RF transmitter adjustment-optimized for patient imaging — adjusts to a high voltage value, which is caused by water's dielectric properties, leading to 2 effects. On the one hand, the magnetization is flipped to such an extent that signal voids can be seen in ring patterns (Fig. 5, row 1, adjusted at $279.5 \mathrm{~V}$ ). On the other hand, artifacts of a different pattern can be observed in the AC in-phase images (Fig. 5, second to last column) and assigned attenuation values (Fig. 5, last column). The latter artifacts derive from the combination of water's high permittivity and its long T1 relaxation time.

Reducing the initial voltage of the adjustment algorithm leads to a lower adjusted RF transmitter voltage and results in the images shown in Figure 5 (row 2, adjusted at $74.6 \mathrm{~V}$ ). The in-phase images of the AC sequence are improved, resulting in an improved AC $\mu$-map. Here, the buildup of a steady state before readout will prevent artifacts in the phase-encoding direction. For this purpose, prescans performed directly before the measurement can lead to the desired result.

The AC $\mu$-maps can also be improved by adding $\mathrm{NiSO}_{4}$ to water. However, in the standard MR protocols (first 3 columns), water plus $\mathrm{NiSO}_{4}$ remains with strong $\mathrm{B}_{1}$ artifacts, visible as central brightening. But the signal intensity in the images is enhanced, and because the T1 time of the fluid stays clearly below typical $\mathrm{T} 1$ values in the human torso (T1 time of, for example, the human liver at $3.0 \mathrm{~T}: 809 \pm 71 \mathrm{~ms})(25)$, the use of default scanner protocols, optimized for patient imaging, is improved. Consequently, a $\mathrm{T} 1$ reduction should generally be preferred when scanning water.

The increased $\sigma$ by the subsequent addition of $\mathrm{NaCl}$ led to a reduction of the central brightening effect in the images (Fig. 5, row 4).

Figure 6 shows the distribution of the $\mathrm{B}_{1}$ field in the fluids and the deviation from the excited $90^{\circ}$ flip angle along the vertical center line in the phantom. The flip angle distribution was further quantified by evaluating ROIs in the outer region and in the center of these $B_{1}$ maps of the phantom (Supplemental Fig.1; supplemental materials are available online only at http://jnm.snmjournals.org). The relation between the mean flip angle in the center of the phantom and the mean flip angle in the outer regions was calculated.

The graphs demonstrate that, compared to pure water, the increased $\sigma$ by the addition of $\mathrm{NiSO}_{4}$ and $\mathrm{NaCl}$ reduces the $\mathrm{B}_{1}$ elevation in the phantom's center to a certain extent (remaining flip angle increase in phantom center by a factor of 1.17). But because the 2 competing effects are not opposed exactly, the RF field cannot be homogenized completely in the phantom. Furthermore, the RF energy deposition increases, causing specific absorption rate (SAR) limits to be reached earlier and thus leading to reduced flexibility in the imaging protocols. Additionally, the generated AC $\mu$-map may be geometrically distorted because of false segmentation of the underlying segmentation algorithm when water is used (Fig. 5, AC $\mu$-map of water plus $\mathrm{NiSO}_{4}$ plus $\mathrm{NaCl}$ ). Monoethylene and triethylene glycol significantly improve RF homogeneity compared to water but exhibit residual minor flip angle elevations in the phantom center for the tested body phantom (flip angle increase of 1.28 and 1.13; Fig. 6). Because of this, the central brightening effect cannot be completely eliminated in the MR images (Fig. 5). The chosen emulsion provides a more homogeneous flip angle distribution (flip angle increase by a factor of 1.06), closer to pure oil. However, as mentioned, resulting air bubbles within the emulsion can be seen in both PET (Fig. 3) and MR images (Fig. 5). 


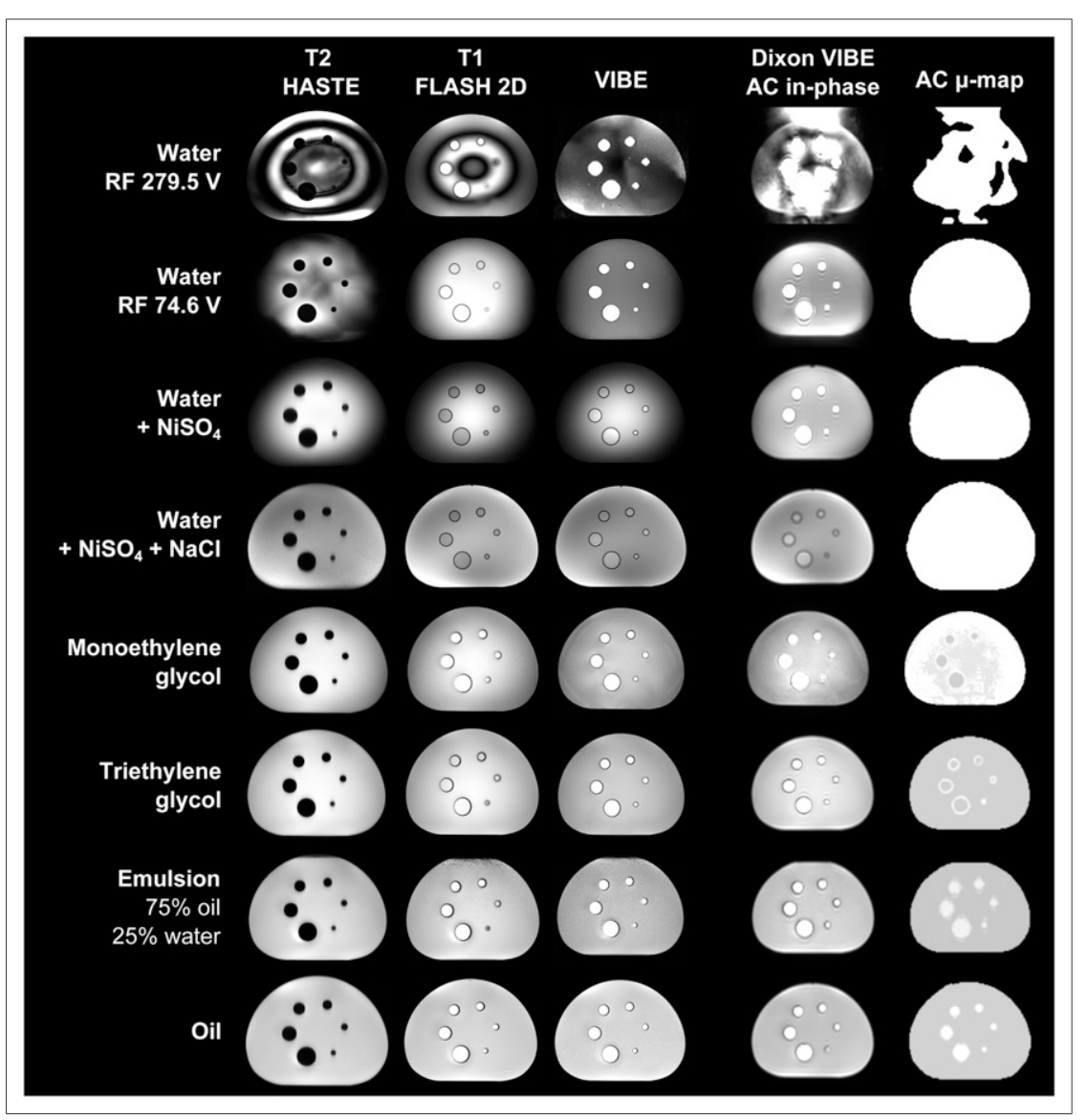

FIGURE 5. Different MR sequences measured with various fluids in PET standard phantom. Inhomogeneous $\mathrm{B}_{1}$ excitation, due to high $\varepsilon_{\mathrm{r}}$ and low $\sigma$, was observed when water was used as phantom fluid (upper 3 rows). Image-windowing properties were adjusted individually for best demonstration purposes. AC $\mu$-maps provide attenuation values of phantom fluid for water, fat, and air only. FLASH = fast low angle shot; HASTE = half Fourier-acquired single-shot turbo spin echo.

\section{Rating Based on Fluid Suitability for ${ }^{18}$ F-FDG PET/MR Measurements}

On the basis of the obtained results, the tested fluids were rated with respect to their suitability for PET/MR phantom measurements. This rating considers PET imaging with ${ }^{18} \mathrm{~F}-\mathrm{FDG}$ as a tracer only. The criteria for this rating are the ability to dissolve ${ }^{18} \mathrm{~F}-$ FDG; homogeneity of RF excitation at $3 \mathrm{~T}$; and a general rating on practicability for phantom experiments, considering aspects such as handling effort (mixing and cleaning), availability, stability, cost, safety, and effort of disposal (Table 4).

Before using the described substances, material safety data sheets for all fluids, including risk and safety statements, need to be considered. $\mathrm{NiSO}_{4}$, for example, is reported to be a carcinogen and as being toxic by inhalation and ingestion. Monoethylene and triethylene glycol exhibit specific target organ toxicity and are an eye and skin irritant as well. Substances should be handled with extra caution.

More cleaning effort is required when substances other than pure water are used. Costs will be highest when glycols are used (at present, $\sim$ \$300 USD per $10 \mathrm{~L}$ of triethylene glycol; costs vary between manufacturers). Prices for all other fluids were below \$50 USD.

\section{DISCUSSION}

In this study, different approaches of phantom fluid and tracer selection were considered and systematically evaluated with regard to their usability for simultaneous PET/MR phantom imaging.

Compared to water, both triethylene glycol and monoethylene glycol improve MR homogeneity notably and therefore enable phantom imaging, even with RFintensive $\mathrm{MR}$ sequences in the given phantom and in phantoms of comparable size. The emulsion was superior in terms of homogeneity of RF excitation in MR imaging; however, its preparation is timeconsuming and cumbersome. Additionally, air bubbles may appear within the emulsion and impair potential quantitative phantom measurements. A different emulsifier composition might exist that yields a more suitable emulsion with better stability and homogeneity. However, the question is whether the advantage of a rather small improvement in $\mathrm{B}_{1}$ homogeneity, compared to triethylene glycol, outweighs the drawback in emulsion preparation.

As mentioned, the mixing process of the tracer and alternative fluid in the large phantom is more time-consuming, and more effort is needed than for the process with water. The best solubility was achieved when the tracer was injected into a small volume of the fluid first, to allow stirring before the tracer-fluid mixture was added to the already partially filled phantom. For increased mixing effectiveness, it is recommended that the phantom not be completely filled at first. Sufficient time (e.g., $45 \mathrm{~min}$ ) should be invested for the tracer to dissolve before the first scan. Other mixing techniques, such as stirring the tracer-fluid mixture in the half-filled body phantom, may eventually lead to an even faster tracer distribution.

Concerning the fluid compatibility with the acrylic glass material of the phantom, this study relied on information obtained from literature (16) or from manufacturers' specifications. The effect of the fluid on additional materials of the acrylic phantom, such as rubber seals or glue, was not considered but should be additionally investigated when long-term exposure to the fluid is planned. In terms of the exposure only for the time of a single or several subsequent measurements, none of these factors limited the use of the proposed substances in the performed scans.

Crown ethers such as used in the ${ }^{18}$ F-fluoride Kryptofix 222 complex showed promising results with their ability to dissolve the tracer in both oil and water. However, in its tested composition, the tracer ${ }^{18}$ F-fluoride Kryptofix 222 is not yet usable for phantom scans. Further preliminary tests suggested that reduced solubility of acetonitrile in oil may cause the problem. Possibly the substitution of acetonitrile in the tracer solution by a different organic solvent, such as dimethylformamide, might enable sufficient solubility in oil. Using azeotropic drying in the tracer synthesis process would clear the tracer solution of water, which may also have negatively affected the solubility in the reported scans. These modifications might enable the use of fluoride- 18 in combination with crown ethers as a tracer in combined PET/MR phantom 


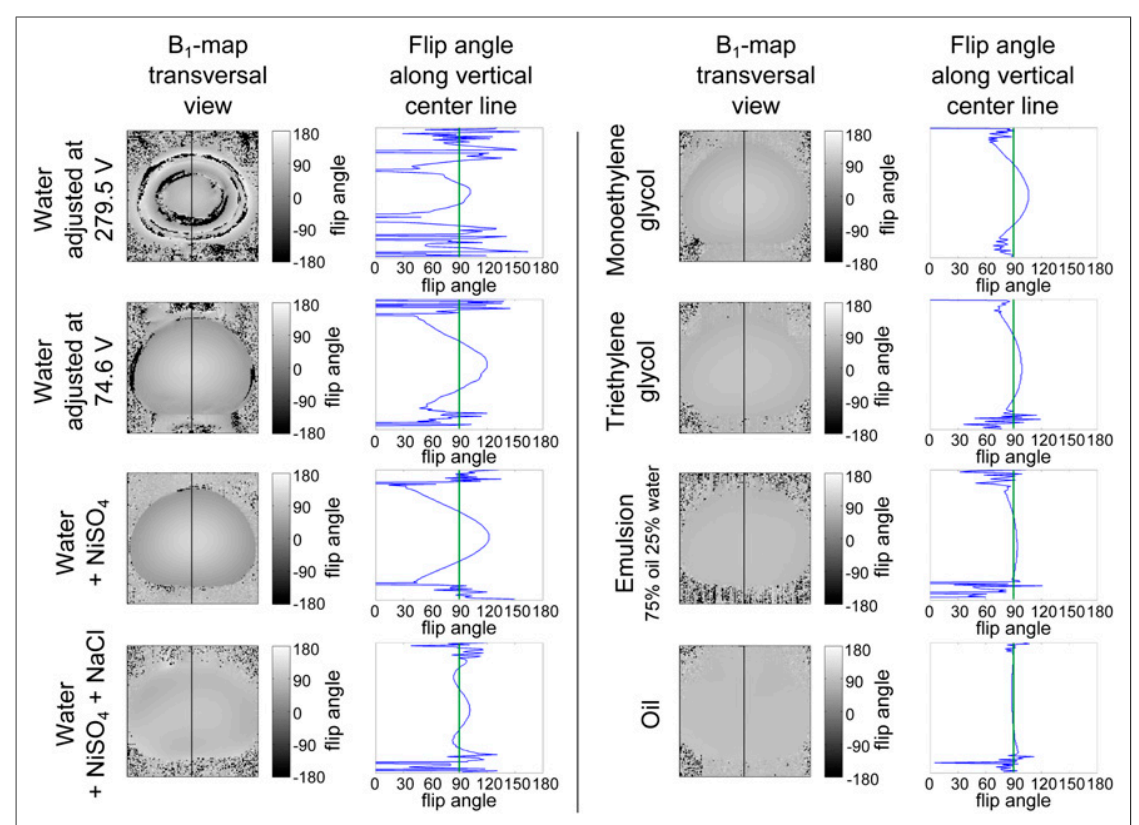

FIGURE 6. Measurement of $B_{1}$ homogeneity as function of phantom fluid in body standard phantom. $B_{1}$ maps and flip angle profiles along vertical center line in transversal orientation show increasing $B_{1}$ homogeneity when using phantom fluids with decreasing $\varepsilon_{r}$ according to Table 1.

measurements with oil. Here, further investigations concerning the suggested alterations of the tracer composition are needed.

Regarding AC of the PET images, this study discusses only the homogeneous display of the phantom content, for example, as an important prerequisite for successful MR-based AC. Because the PET/MR scanner in the current implementation differentiates only between water and fat attenuation values, the $\mathrm{AC}$ values for other fluids, such as glycols, need to be adjusted manually. Additionally, both phantom plastic housing and glass material of hollow spheres are materials that need to be attenuation-corrected for, to obtain a true PET quantification. The effect of neglecting AC for these materials can be observed in Figure 4. The decrease of activity concentration at the edges of the plots in coronal and transversal

TABLE 4

Rating of Fluids on Criteria Important for PET and MR Imaging

\begin{tabular}{cccc}
\hline Fluid & $\begin{array}{c}\text { RF } \\
\text { solubility } \\
\text { solf }\end{array}$ & $\begin{array}{c}\text { homogeneity } \\
\text { in MR at 3.0 T }\end{array}$ & Practicability \\
\hline $\begin{array}{c}\text { Water } \\
\text { Water }+\mathrm{NiSO}_{4}\end{array}$ & +++ & --- & +++ \\
$\begin{array}{c}\text { Water }+\mathrm{NiSO} \\
+\mathrm{NaCl}\end{array}$ & +++ & --- & - \\
$\begin{array}{c}\text { Monoethylene } \\
\text { glycol }\end{array}$ & + & + & - \\
$\begin{array}{c}\text { Triethylene } \\
\text { glycol }\end{array}$ & + & + & + \\
Emulsion & & ++ & + \\
Oil & + & +++ & - \\
\hline
\end{tabular}

+++ = very good; - - - = not acceptable; - = complex handling; $+=$ sufficient; $++=$ good. orientations may be caused by missing AC of the phantom's plastic housing, the glass spheres, and, additionally, the flexible body matrix coil placed on top. Especially in the front part of the phantom, in transversal orientation, these effects sum up because of the thickness of the plastic housing and the presence of an RF cable trap (RF balun) in the flexible body matrix coil (26). Preliminary experiments testing the use of ultrashort-echo time sequences for an MR-based depiction of the plastic housing (data not shown) resulted in insufficient phantom signal and critical image quality, which did not allow for an adequate assessment of the phantom housing at present.

In this study, triethylene glycol remains the best alternative to water and oil. Finally, the choice of fluid depends on the desired application. If only a homogeneous attenuation map of the fluid-filled regions is required, the use of water scanned with reduced transmitter voltage or water with added $\mathrm{NiSO}_{4}$ may lead to the desired result using the current AC-scanning parameter settings. Nevertheless, to obtain full flexibility in the choice of imaging parameters and for homogeneous MR contrast throughout the spectrum of MR sequences, an alternative fluid to water is mandatory.

\section{CONCLUSION}

This study provides a systematic approach of phantom fluid selection for a given quality-standard phantom - and phantoms of comparable size-at 3.0 T MR field strength. For simultaneous PET/MR phantom scans using the standard tracer ${ }^{18} \mathrm{~F}-\mathrm{FDG}$, triethylene glycol as an alternative fluid to water and oil is proposed that serves as a viable option for both PET and MR imaging. When water is preferred, workarounds for generating an improved AC $\mu$-map are presented.

An additionally tested PET tracer, ${ }^{18}$ F-fluoride Kryptofix 222 complex, enables the use of pure oil in simultaneous PET/MR scans; however, the tracer needs to be optimized for the application in phantom measurements.

\section{DISCLOSURE}

The costs of publication of this article were defrayed in part by the payment of page charges. Therefore, and solely to indicate this fact, this article is hereby marked "advertisement" in accordance with 18 USC section 1734. The Biograph mMR PET/MR system at the IMP was funded through a research collaboration between Siemens Healthcare Sector, Erlangen, Germany, and the University of Erlangen-Nuremberg. No other potential conflict of interest relevant to this article was reported.

\section{ACKNOWLEDGMENTS}

We thank Ingo Weiss, $\mathrm{PhD}$, and Heinrich Büssing, $\mathrm{PhD}$, from Biotronik SE \& Co. KG, Berlin, Germany, for performing measurements and providing data about permittivity and conductivity 
of the fluids. Also, we thank Ralf Ladebeck (Siemens Healthcare Sector, Erlangen, Germany) for his constant support and time for many discussions along the course of this project. Jens U. Krause, Institute of Medical Physics, University of Erlangen-Nuremberg, Erlangen, Germany, is acknowledged for tracer handling when PET measurements were performed. Additionally Bharath Navalpakkam, Pattern Recognition Lab, University of ErlangenNuremberg, Erlangen, Germany, is thanked for editorial support.

\section{REFERENCES}

1. Zaidi H, Ojha N, Morich M, et al. Design and performance evaluation of a whole-body Ingenuity TF PET-MR imaging system. Phys Med Biol. 2011;56:3091-3106.

2. Delso G, Fürst S, Jakoby B, et al. Performance measurements of the Siemens mMR integrated whole-body PET/MR scanner. J Nucl Med. 2011;52: 1914-1922.

3. Bernstein MA, Huston J, Ward HA. Imaging artifacts at 3.0 T. J Magn Reson Imaging. 2006;24:735-746.

4. Collins CM, Liu W, Schreiber W, Yang QX, Smith MB. Central brightening due to constructive interference with, without, and despite dielectric resonance. J Magn Reson Imaging. 2005;21:192-196.

5. Ladd ME. High-field-strength magnetic resonance: potential and limits. Top Magn Reson Imaging. 2007;18:139-152.

6. Sled JG, Pike GB. Standing-wave and RF penetration artifacts caused by elliptic geometry: an electrodynamic analysis of MR imaging. IEEE Trans Med Imaging. 1998; 17:653-662.

7. Ibrahim TS, Lee R, Abduljalil AM, Baertlein BA, Robitaille P-ML. Dielectric resonances and B1 field inhomogeneity in UHFMRI: computational analysis and experimental findings. Magn Reson Imaging. 2001;19:219-226.

8. Vaidya MV, Sodickson DK, Brown R, Wiggins GC, Lattanzi R. B1+ and B1field pattern dependence on the electrical properties of the sample and the static magnetic field strength [abstract]. Proc Intl Soc Mag Reson Med 20. 2012:2796.

9. Merkle EM, Dale BM. Abdominal MR imaging at $3.0 \mathrm{~T}$ : the basics revisited. AJR. 2006;186:1524-1532.

10. Tofts PS. Standing waves in uniform water phantoms. J Magn Reson B. 1994;104:143-147.
11. Simmons A, Tofts PS, Barker GJ, Arridge SR. Sources of intensity nonuniformity in spin echo images at 1.5 T. Magn Reson Med. 1994;32:121-128.

12. Skloss T. Phantom fluids for high field MR imaging [abstract]. Proc Intl Soc Magn Reson Med 11. 2004:1635.

13. Pichler BJ, Judenhofer MS, Catana C, et al. Performance test of an LSO-APD detector in a 7-T MR imaging scanner for simultaneous PET/MR imaging. $\mathrm{J}$ Nucl Med. 2006;47:639-647.

14. International Electrotechnical Commission (IEC). Radionuclide Imaging Devices: Characteristics and Test Conditions-Part 1: Positron Emission Tomographs. Geneva, Switzerland: IEC; 1998:27-28.

15. National Electrical Manufacturers Association. NEMA Standards Publication NU 2-2007. Performance Measurements of Positron Emission Tomographs. Rosslyn, VA: NEMA; 2007:26-33.

16. Plastics Design Library. Chemical Resistance, Volume I: Thermoplastics. Morris, NY: Plastics Design Library; 1994:81.

17. Kotz JC, Treichel PM, Townsend J. Chemistry \& Chemical Reactivity. Belmont, CA: Brooks/Cole; 2009:560.

18. Brown WH, Foote CS, Iverson BL, Anslyn EV. Organic Chemistry. Belmont, CA: Brooks/Cole; 2012:458-459.

19. Yu S. Review of ${ }^{18}$ F-FDG synthesis and quality control. Biomed Imaging Interv J. 2006;2:e57.

20. Hamacher K, Coenen HH, Stöcklin G. Efficient stereospecific synthesis of nocarrier-added 2-( $\left.{ }^{18} \mathrm{~F}\right)$-fluoro-2-deoxy-D-glucose using aminopolyether supported nucleophilic substitution. J Nucl Med. 1986;27:235-238.

21. Saha GB. Basics of PET Imaging: Physics, Chemistry and Regulations. New York, NY: Springer; 2010:132-133.

22. Schlyer DJ. PET tracers and radiochemistry. Ann Acad Med Singapore. 2004; 33:146-154.

23. Biersack H-J, Freeman LM. Clinical Nuclear Medicine. Berlin, Germany: Springer-Verlag; 2007:67.

24. Martinez-Möller A, Souvatzoglou M, Delso G, et al. Tissue classification as a potential approach for attenuation correction in whole-body PET/MR imaging. J Nucl Med. 2009;50:520-526.

25. de Bazelaire CMJ, Duhamel GD, Rofsky NM, Alsop DC. MR imaging relaxation times of abdominal and pelvic tissues measured in vivo at $3.0 \mathrm{~T}$ : preliminary results. Radiology. 2004;230:652-659.

26. Paulus DH, Braun H, Aklan B, Quick HH. Simultaneous PET/MR imaging: MRbased attenuation correction of local radiofrequency surface coils. Med Phys. 2012;39:4306-4315. 